\title{
Analysis on the Surgery Effect Observation of the Blunt from Middle Finger Replantation
}

\author{
Xin Wang ${ }^{1, a}$, Yan Zhao ${ }^{1, *}$, Yeben Wang ${ }^{1}$, Liang Zhao ${ }^{1}$ \\ ${ }^{1}$ Department of Department of, The third People's Hospital of Jinan, Jinan 250132, China \\ awangxin@126.com \\ *Corresponding author zhaoyan@163.com
}

Keywords: Blunt from middle finger; Reattachment; Repair

\begin{abstract}
Objective: To observe and investigate the surgery effect of the blunt from middle finger replantation. Methods: We select from August 2012 to August 2012 and given surgical treatment of 50 patients with blunt from middle finger, all patients were given microscopic reattachment surgery the survival rate of replantation surgery. Result: 45 patients in 50 cases with postoperative replantation are survival, the survival rate is $90.00 \% ; 5$ patients after replantation failure, failure rate is $10.00 \%, 5$ cases of replantation failure patients, 2 cases of patients with postoperative infection and cause the failure of replantation, 3 patients caused by vascular crisis occurred after replantation and the distal finger necrosis and failure. Conclusions: In reattachment surgery for patients with thorough debridement, precise vascular anastomosis, it is careful to repair finger organization, effective prevention of vascular crisis is critical to the success of the surgery, and is worth clinical promotion.
\end{abstract}

\section{Introduction}

The severed finger is one of clinical common serious injury, not only bring pain to patients, and if they can't get timely and effective treatment, on the one hand, will cause the injured finger dysfunction, are more likely to affect the mental health of the injured man on the other hand, bring to life after the shadow. Replantation is refers to under the magnifying glass (microscope), will be away from the fault refers to the weight of the new replantation in situ, through the nerve anastomosis, restore blood circulation and the fingers function operation. With medical progress, especially the development of microsurgery technology progress, burned her finger replantation has been widely. However, because of the surgery itself high technical requirements, with complicated injury, infection, blood vessel crisis and other factors, clinical still nearly $10 \%$ of the patients with surgical failures and causes damage, therefore, to explore the clinical effect and its influencing factors of analysis of the operation, will help to enhance the survival rate of replantation of. Patients with avulsion and saw cutting, extrusion and other injuries, finger fracture situation become can cause patients from blunt injury. Traditional treatment methods for such damage does not produce good effect, after finger replantation necrosis or finger function cannot fully recover, seriously affects patients' daily life, reduce the patients quality of life. But in recent decades, with the gradual development of microsurgery technology mature also is widely applied in clinic, and just for the severed finger replantation surgery patients played a major role in the operation is safe and effective, good postoperative finger function recovery, obtained the high praise. In this paper the microstructure of the wounded limb treatment the curative effect of blunt from middle finger for further analysis, pay attention to explore the cause of operation failure and postoperative recovery in patients with poor, relevant improvement measures are discussed. The following specific is reported. 


\section{Materials and Methods}

\section{Materials}

We select from August 2012 to August 2014 were analyzed and surgical treatment of 50 patients with blunt from middle finger, in patients aged between $16 \sim 65$ years, the average age of 37.3 years, including 16 cases of male patients and 4 cases of female patients. All patients were confirmed by the detailed diagnosis for blunt finger was hurt, need reattachment surgery. Patient injury reason, mainly including torn, mechanical damage, squeezing ground injury and saw damage, mainly mechanical ground injury and extrusion damage, including 8 cases of patients as the index finger injury, 5 cases of thumb injury, 3 patients were middle finger injury, as the little finger injury, 2 cases with remaining 1 case for the ring finger injury.

\section{Methods}

In all patients for effective early bleeding and disinfection treatment, after giving microscopic reattachment surgery patients, surgical treatment for the patient first intramuscular injection of scopolamine to $10 \mathrm{mg}$ mountain analgesia and moderate vascular smooth muscle, intravenous drip during the operation of low molecular dextran $500 \mathrm{ml}$ to expand health and optimization of microcirculation, use of a tourniquet was conducted in the patients with upper arm bound to stop bleeding, and for brachial plexus anesthesia. Fingers are away from the broken parts for the section, if patients are given refers to patients with $2 \%$ lidocaine anesthesia. Before and after surgery should also give certain appropriate antispasmodic, anticoagulant and anti-infection treatment. After the osseous rebuilt support, can be appropriate to remove injured bone for stitching, choose wire according to patients' condition, the tools for fixed type needle and so on. After make the joints, ligaments and tendons repairs as well as the patients of vascular anastomosis, muscle function in patients with guarantee and joint stability, and ensure the normal blood supply of finger necrosis. Finally on patients with nerve bundle membrane and the suture without tension, transplant the rest parts of the cutaneous nerve when necessary. According to the condition after the operation, can be in $g$ needle fixed pulled about 10 weeks, and proper rehabilitation exercise. If it is worth noting that the patients suffering from department is located in the joints, refers to the function in patients with retained to a great extent, should try to retain surface of joints.

\section{Results}

The results showed that 50 cases of 45 patients with postoperative replantation is survival, the survival rate is $90.00 \%$; 5 patients after replantation failure, failure rate is $10.00 \%$, 5 cases of replantation failure patients, 2 cases of patients with postoperative infection and cause the failure of replantation, 3 patients caused by vascular crisis occurred after replantation and the distal finger necrosis and failure. See Fig.1 and Fig.2

\section{Discussion}

The blunt from middle finger is refers to the patients suffered a cut, extrusion, avulsion and saws and other damage, caused by finger soft tissue in patients with severe trauma, serious damage to the normal anatomy. And with the rapid development of today's industry, transportation, etc, the harm is also very common in clinic. In current clinical practice, generally uses the reattachment surgery under the microscope, after surgery can restore amputation patients most function, generally does not affect the patients quality of life. This generally includes surgical debridement, internal fixation, muscle ligament repair, vascular anastomosis, refers to the nerve repair, and postoperative function exercise 6 units, may result in any part of the negligence of operation effect of reducing, serious when patients caused by finger necrosis, make the operation failure, therefore in the microscopic limb reattachment, asked the performer's superb skill and patience. Debridement of processing is generally not perfect, the operation is simple and crude caused vascular trauma and infection in patients with sterile and improper application of noninvasive technology can contribute to patients 
with postoperative function is not perfect. The most critical factor is that the precision of anastomosis surgery, should guarantee the elasticity of vascular and light the whole agreement, and as far as possible to reduce patients' vascular tension, once the match is undeserved can cause patients with vascular crisis, insufficient blood supply leading to amputation in patients with distal necrosis and make the operation failed. The visible success rate was $90.0 \%$ in this paper; the treatment is a safe and effective treatment. 5 cases of replantation failure patients, 2 cases of patients with postoperative infection and cause the failure of replantation, 3 patients caused by vascular crisis occurred after replantation and the distal finger necrosis and failure., the error of the aseptic manipulation and vascular anastomosis step is the key to the operation failed, should pay attention to.
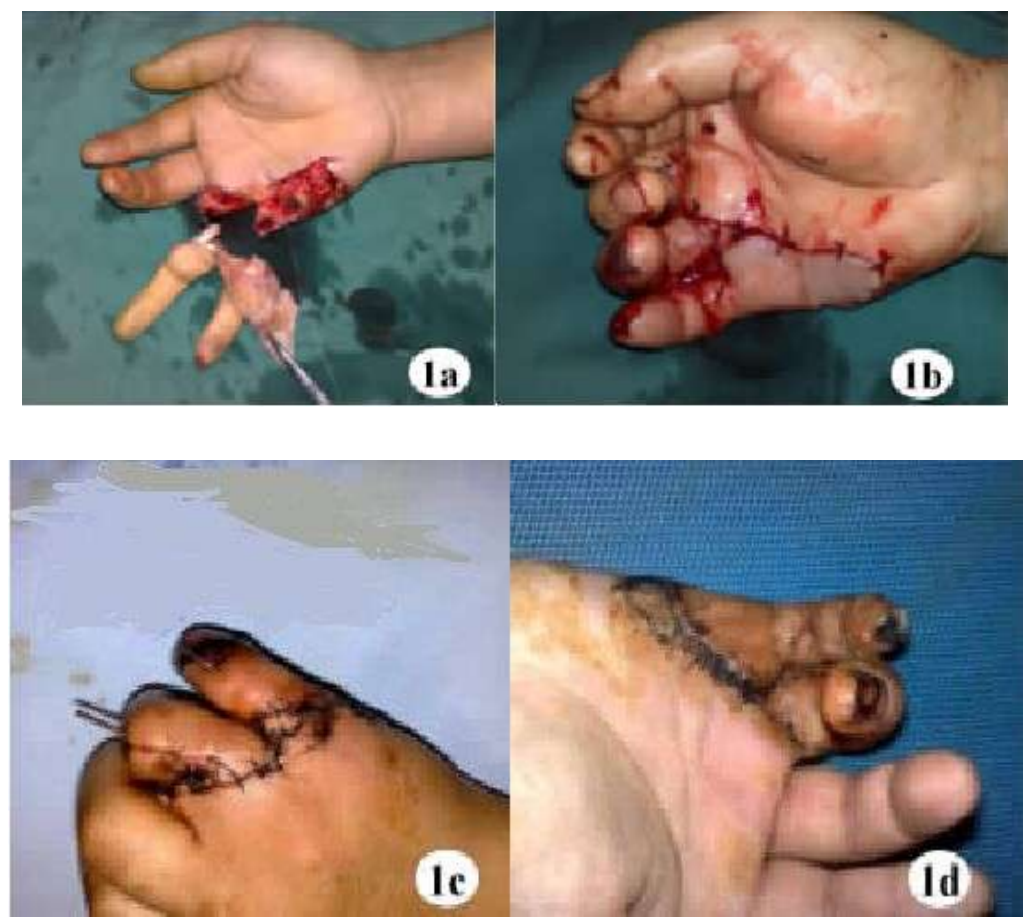

Fig.1 Patient 1: 1a preoperative, $1 \mathrm{~b}$ postoperative, $1 \mathrm{c}$ postoperative after $12 \mathrm{~d}, 1 \mathrm{~d}$ postoperative after 2 months
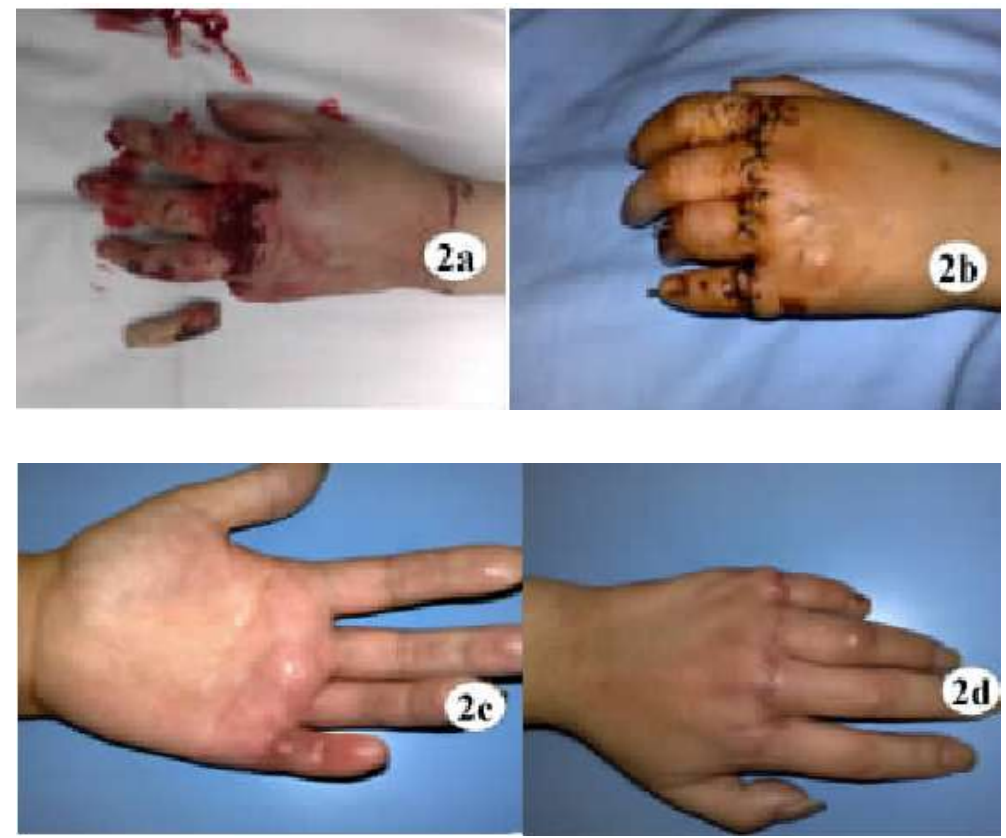

Fig. 2 Patient 2: 2a preoperative, $2 \mathrm{~b}$ postoperative after $14 \mathrm{~d}, 2 \mathrm{c}, 2 \mathrm{~d}$ postoperative after 3 months 
In daily life, due to careless broken injury patients were compared with a common, therefore refers to injury should be broken replantation surgery as soon as possible. Should close observation of postoperative refers to the blood circulation, and recorded at any time. For clinical observation and treatment of finger tip blood circulation, to every 30 to $60 \mathrm{~min}$ to observe one color, the change of temperature and strain, test the capillaries. If the patient's body changed from red to pale, or become a light grey or become a ruddy flower porphyritic, skin temperature starts to drop $3^{\circ} \mathrm{C}$, capillary filling time is extended to 3-4 s stomach tension decreases gradually, suggesting that is artery crisis, we should first adopt antispasmodic and analgesic drug therapy, observe patients after $30 \mathrm{~min}$ is still no improvement, consider immediate surgical exploration. If the patient refers to the body from the red to dark red, and then become a dark purple, skin temperature also gradually fell by $2^{\circ} \mathrm{C}$, capillary filling time $<1 \mathrm{~s}$, stomach tension increased gradually, serious when appear plain, this tip is venous crisis. We must immediately replacement patients around the wound dressings, clean up the wound hemorrhage in time, to dismantle the 1-2 needle incision in patients with sutures, to alleviate venous pressure. If the venous crisis has not ease, feasible or distal lateral pull small incision bleeding. In clinical treatment and care, the disinfection and isolation system is strictly and aseptic technique, to prevent infection. For patients with wound, routine bacterial culture and drug sensitive test should be to choose a more effective antibiotic therapy. Even at the same time enhance the patient's diet and nutrition, effective health education guidance, improve the success rate of patients wounded finger.

The elements of a successful replantation surgery has a lot of, including injury reason and injury, injury after the treatment, treatment time, patients' physical condition, etc. And, of course, the medical staff medical technology is also related to the success rate of surgery. Injury reason and injury is the key to the success of replantation, tissue and blood vessels after injury condition is the foundation of the operation. Therefore, the handling of the original injury should be treated with caution, should not be the mesh. The injured should be timely medical treatment. Studies have shown that heat ischemia within $12 \mathrm{~h}$ don't have a significant impact on about the success rate of surgery, and after $12 \mathrm{~h}$, the longer the time, the lower the survival rate. In general, should not be more than $24 \mathrm{~h}$. The longer the severed finger ischemia time, the degree of tissue necrosis, even restore blood circulation, reperfusion injury may occur. Combined with clinical cases clinically, according to the normal anatomical characteristics of the fingers, has carried on the classification to amputation. The patient's condition and operation are important to the success of the relationship. Vascular anastomosis is replantation is one of the key to survival, should as far as possible consistent with the contra lateral artery and vein, as much as possible in order to reduce the probability of anastomosis failure. For artery, common operation method is end to end anastomosis, very conditions, can also be transplanted, but demand for microsurgical technique is quite high. Direct vein end to end anastomosis, vein transplantation can rebuild the blood circulation. For the distal finger, it can do a pull and incision bleeding maintain blood circulation Agreement with the progress of technology, 0.2-0.4 $\mathrm{mm}$ small blood vessels has become the normal operation, also makes the indication of replantation is constantly expanding, the appearance of the finger at the same time, the recovery of function can be better guaranteed.

The complete debridement is the basis of replantation is successful, is the implementation of replantation of prerequisites, elaborate finger organization, only the naked eye is hard to distinguish the organization degree of pollution and contusion, debridement under a microscope, not only reduce the chance of infection, but also create conditions for tissue repair. Debridement and detection refers to the blood vessels, nerves, completes the tag, is advantageous for the finger to find and match after reset. Vascular anastomosis operation stability, accurate, lightweight, smart, for anastomosis arteriovenous order, our group have to fit a first refers to the inherent artery, loosen the blood with 5-10 min, see if can provide the blood supply of severed fingers (that is, the blood supply is obstructed), vascular remaining a little blood clot and part with venous oxygen metabolites discharge, and then match venous reflux is established. For arteriovenous length is insufficient, can be more blood vessels along the length, shortening phalange reduce vascular 
tension, such as when necessary in near the finger or forearm shallow vascular remodeling. Intraoperative for tendon defect, our group has a long period of palm tendon graft reconstruction in 3 cases, the first phase of reconstruction meet early postoperative functional training, the author choose a while and vascular bed condition is good, high replantation opportunity refers to cancer, and for a while and vascular bed condition is bad, all the survival after replantation of secondary processing tendons, need to communicate with patients preoperatively.

Nerve repair is very important to restore the sense and its functions of replantation of amputated, generally for end to end anastomosis, porting when necessary. For patients with postoperative antibacterial, anticoagulant, antispasmodic treatment, the antibiotics postoperatively is for 3-5 d antibacterial treatment. Opium poppy alkaloid can be used for antispasmodic, but should be used in the injection site, prevent fluids mass. Although whether postoperative systemic application of anticoagulant drugs remains controversial, but foreign scholars are still in use, think anticoagulants can reduce anastomosis of blood clots. The multi-purpose small molecule dextran at present, drugs are such as aspirin. Of course, if blood vessels are badly damaged or found thrombosis, vascular transplantation, should be careful application of anticoagulant therapy. Vascular crisis is one of important postoperative complications, and the first three days after surgery is the easiest. Should pay close attention to the patient's postoperative circulation situation of severed fingers, such as the color of finger replantation, temperature, and stomach, capillary filling test, etc., if the fingers appear pale, gray, blue, such as vascular crisis, should be timely and accurate judgment for processing, in order to improve the survival rate of finger replantation. Then vascular crisis, venous crisis is common, can use $12500 \mathrm{u}$ add in $12500 \mathrm{ml}$ of heparin saline, wash the wound every hour, and promote circulation.

The rational is use of hyperbaric oxygen to patients. In patients with hyperbaric oxygen, around the small blood vessels, lead to peripheral circulation resistance increases, reduced the amount of tissue perfusion, patients benefit. However, patients with decompression, can appear rebound phenomenon, and compound tissue ischemia hypoxia or even cause seizures. It has been suggested that in the patients with replantation is early, no application of hyperbaric oxygen therapy. Good communication with patients and their families, for patient to cooperate, to strengthen the fingers function exercise, to restore finger function has a good effect. Generally in about three weeks after the surgery, into the soft tissue healing period, amputation patients should cooperate with slight flexion and finger movement, 30-50 times/day. 4-6 weeks postoperatively, if bone connection is good, should be more proper massage, proper length contraction exercises, such as clip sandbags (50-100 - g/sandbags); After six weeks, should be the mouth often activities to promote the recovery of neural function.

\section{Conclusion}

In conclusion, the patients with a thorough debridement, precise vascular anastomosis, carefully repair of finger organization; effective prevention of vascular crisis in the reattachment surgery is the key to successful operation. For the patients with blunt from middle finger, the microstructure of the wounded limb is a safe and effective treatment, the prevention of surgical infection and vascular anastomosis of precision or not is the key to the operation; the treatment is worth further promotion.

\section{Acknowledgement}

This research was financially supported by the National Science Foundation.

\section{References}

[1] Andreasveihelmann, Anthony Gustaveharris, Fritzkrombach, Elkeschütze, Hans Jürgenrefior, Konradmessmer. In Vivo Assessment Of Synovial Microcirculation And Leukocyte - Endothelial Cell Interaction In Mouse Antigen - Induced Arthritis[J]. Microcirculation. 2012 (4) 
[2] H.-J. Anders,C. Haedecke, T. Sigl,K. Krüger. Avascular Areas on Nailfold Capillary Microscopy of Patients with Wegener's Granulomatosis[J]. Clinical Rheumatology . 2013(2)

[3] Ernst von Dobschuetz,Tomas Hoffmann,Konrad Messmer. Diaspirin Cross-linked Hemoglobin Effectively Restores Pancreatic Microcirculatory Failure in Hemorrhagic Shock[J]. Anesthesiology. 2012 (6)

[4] Brian D Duscha,William E Kraus, Steven J Keteyian, Martin J Sullivan,Howard J Green, Fred H Schachat, Anne M Pippen, Clinton A Brawner, Jason M Blank, Brian H Annex. Capillary density of skeletal muscle[J]. Journal of the American College of Cardiology. 2012(7)

[5] Marc S Rendell, Mary F Finnegan, Thomas Pisarri, James C Healy, Anne Lind,Brian K Milliken, David E Finney, Robert F Bonner. A comparison of the cutaneous microvascular properties of the Spontaneously Hypertensive rat and the Wistar-Kyoto rat[J]. Comparative Biochemistry and Physiology, Part A. 2013 (4)

[6] Tarek F.T. Antonios,Donald R.J. Singer, Nirmala D. Markandu,Peter S. Mortimer,Graham A. MacGregor. Structural Skin Capillary Rarefaction in Essential Hypertension[J]. Hypertension. $2012(4)$

[7] Ellen Jendraschak,E. Helene Sage. Regulation of angiogenesis by SPARC and angiostatin: implications for tumor cell biology[J]. Seminars in Cancer Biology. 2013 (3)

[8] Plate KH,Breier G,Millauer B,et al.Up-regulation of vascular endothelial growth factor and its cognate receptors in a rat glioma model of tumor angiogenesis. Cancer Research. 2014(5).

[9] Asahara T,Murohara T,Sullivan A,et al. Isolation of putative progenitor endothelial cell for angiogenesis. Science. 2013(6).

[10] Diaz-Flores L,Gutierrez R,Varela H. Angiogenesis: an update. Histology and Histopathology. 2013(2).

[11] Jendraschad B, Sage BH. Regulation of angiogenesis by SPARC and angiostatin: implications for tumor cell biology. Seminars in Cancer Biology. 2012(8). 